\title{
Method for Detection of Bacteriuria Suitable for Use in General Practice
}

\author{
J. M. BRADLEY,* M.B., B.S. ; N. CROWLEY, † M.R.C.S., L.R.C.P., F.C.PATH. \\ J. H. DARRELL, $\ddagger$ M.B., D.C.H., M.C.PATH.
}

Brit. med.F., 1967, 4, 649-651

A completely satisfactory screening method for bacteriuria requiring the minimum of apparatus and bench expertise and suitable for use in general practice has still to be described. Even where laboratory facilities are readily available to general practitioners transport of the specimen without the delay which would upset the quantitative aspects of the investigation is often impossible. In addition, hospital laboratories would be unable to cope with the demands that ought to be made on them if all diagnosed urinary tract infections were adequately followed. The investigation of patients whose symptoms are less conclusive but who nevertheless may have urinary tract infections (Mond et al., 1965) would add considerably to the volume of work.

A method employing disposable commercial swabs to inoculate MacConkey plates that has been developed and used for the screening of antenatal patients for significant bacteriuria at the Royal Free Hospital overcomes these disadvantages. The method has been employed without difficulty in the clinic by nursing staff, in general practice, and by medical students.

\section{Methods}

The Swab.-Pre-sterilized disposable commercially available swabs having a tightly wound cotton-wool tip $1 \mathrm{~cm}$. in length were used to inoculate the medium. A machine-made swab was selected to minimize variation in size.

The Test.-Fresh preferably clean voided specimens were obtained in sterile wide-mouthed jars. In all cases specimens were plated by immersing the entire cotton-wool tip in the urine and briefly draining it against the inside of the jar before

- Senior Lecturer, Department of Microbiology, Royal Free Hospital School of Medicine, London W.C.1.

t Reader in Bacteriology, Royal Free Hospital School of Medicine, London W.C.1.

$\ddagger$ Lecturer, Department of Clinical Bacteriology, Royal Postgraduate Medical School, London W.12. using it to inoculate a single dried MacConkey plate. An inoculum of four parallel blocks, each comprising four strokes of the swab, was made without rotating the swab (Fig. 1).

Variation in Uptake of Urine by the Swabs.Twenty swabs were weighed before and after charging with urine to discover the variation in absorbency.

Correlation between Swab Plating, Standard Loop Inocula, and Viable Counts. -With the use of a technique modified from that of Miles and Misra (Cruickshank, 1965) viable counts were done on 20 specimens which were also plated by the swab method. In addi-

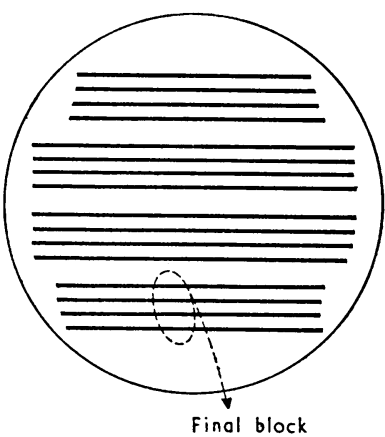

Fig. 1.-Pattern of swab inoculum tion approximately 500 specimens were plated by the swab and standard loop (Guttmann and Stokes, 1963) methods. The standard loop method had been extensively compared with viable counts when it was first adopted in this laboratory for the routine culture of urine specimens six years ago (unpublished observations). Twenty-six further specimens were examined by both the standard loop and Miles and Misra methods.

Incubation.-Cultures were routinely incubated at $37^{\circ} \mathrm{C}$. One hundred specimens were inoculated in duplicate by the swab method, and the duplicate plates were held at room temperature $\left(17-22^{\circ}\right.$ C. $)$. Another 26 specimens were held at $4^{\circ} \mathrm{C}$. for up to 120 hours before incubation at $37^{\circ} \mathrm{C}$.

\section{Results}

The Test.-By not rotating the swab during plating a slight progressive dilution was obtained. The final block inoculated

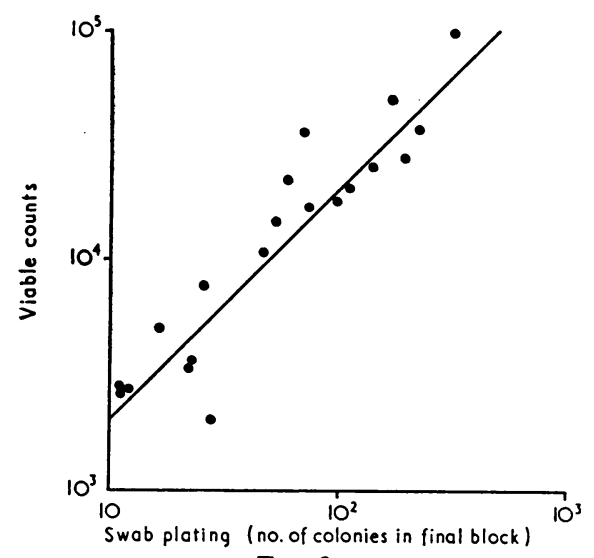

FIG. 2

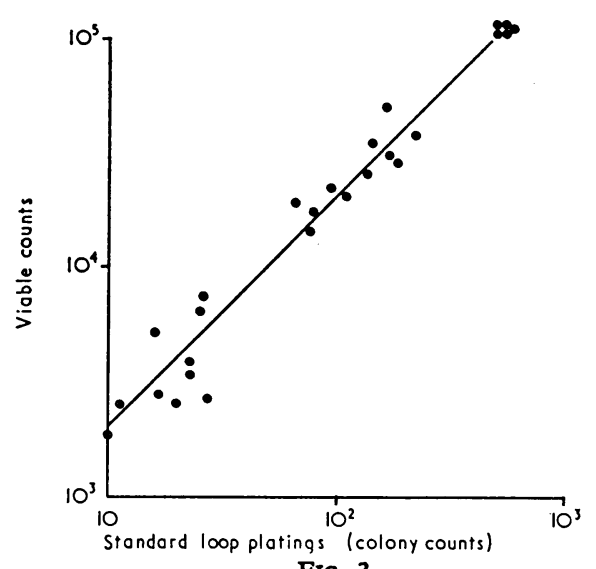

Frg. 3

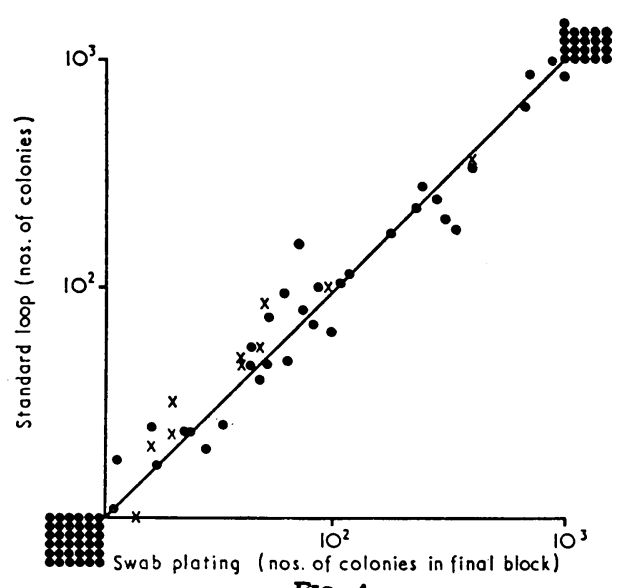

FIG. 4

FIG. 2.-Comparison between numbers of organisms per ml. of urine calculated by the Miles and Misra method and colony counts of the final blocks of swab platings. FIG. 3.-Comparison between numbers of organisms per ml. of urine calculated by the Miles and Misra method and colony counts of standard loop platings. FIG. 4.- Comparison between colony counts of standard loop platings and the final block of swab platings. Crosses represent infections with Gram-positive cocci, and dots infection with Gram-negative rods. The unrealistic number of specimens in the 50-500 colonies per inoculum range is due to selection of suitable specimens for comparison. 
was used to read the test, and, following our usual practice, colonies were counted up to about 300 colonies per inoculum and estimated above this.

Variation in Uptake of Urine.-The weights of urine absorbed by the 20 swabs ranged between 0.1106 and $0.1441 \mathrm{~g}$., with a mean of $0.1262 \mathrm{~g}$. and a standard deviation of $0.0115 \mathrm{~g}$. Three weighings differed from the mean by more than one standard deviation.

Correlations.-Fig. 2 shows the correlation between the swabplating method and the viable counts done on 20 specimens. Fig. 3 shows the correlation between the standard loop plating method and the viable counts done on 26 specimens. Fig. 4 shows the correlation between the numbers of colonies in the last block of each swab plating and the standard loop method for 102 specimens collected and plated in general practice and further investigated in the hospital laboratory. Similar results were obtained with about 400 more specimens treated this way in hospital. Figs. 5 and 6 show standard loop and the corresponding swab platings. It has also been the experience of one of us (N.C.), when using similar swabs to make subcultures from liquid media, that it is practically impossible to overload the swabs, or to vary the amount of fluid absorbed, by alterations in the contact time between swab and fluid medium. The swabs are exceptionally absorbent and are fully loaded instantly.

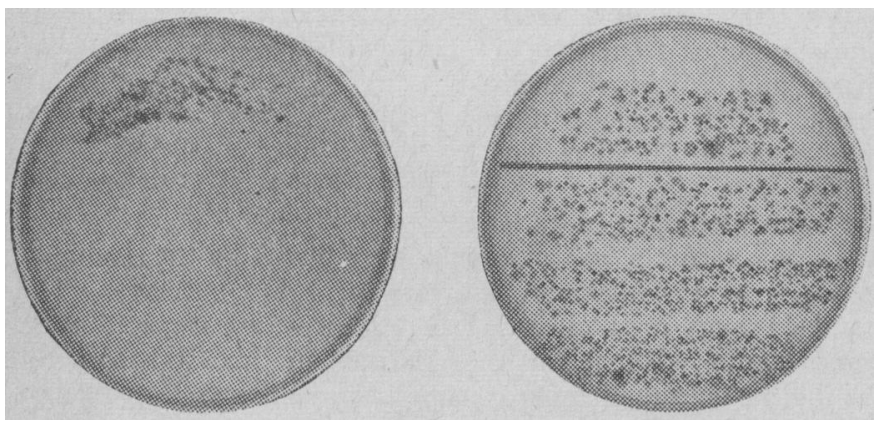

FIG. 5.-Standard loop and corresponding swab inoculum of an insignificant mixed growth of two types of lactose-fermenting organisms.

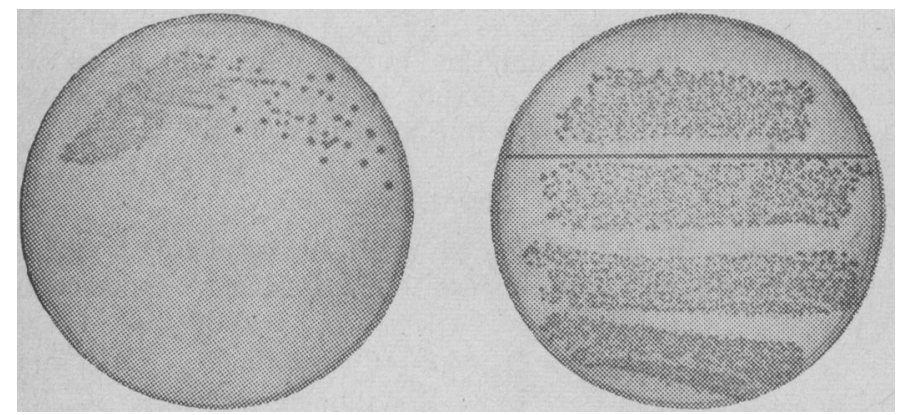

FIG. 6.-Standard loop and corresponding swab inoculum of a significant growth.

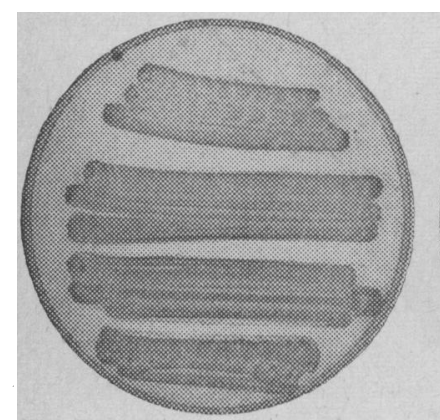

R.T.

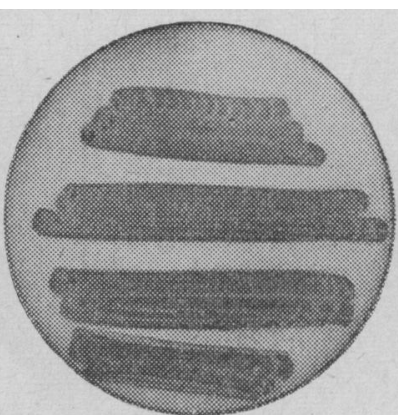

$37^{\circ} \mathrm{C}$.

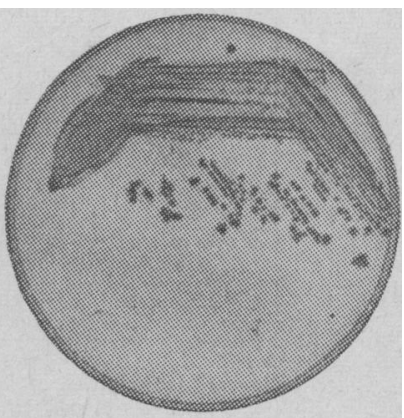

$37^{\circ} \mathrm{C}$.
FIG. 7.-Comparison between urine culture inoculated by swab and standard loop methods and incubated at $37^{\circ}$ C. overnight, and inoculated by swab method and retained at room temperature (approximately $21^{\circ}$ C.) for 48 , hours. The organism was Escherichia coli.
Incubation.-Though in routine practice plates were incubated at $37^{\circ} \mathrm{C}$. overnight, plates held at room temperature overnight were often readable, and, apart from one Proteus species which did not grow at room temperature, all members of the Enterobacteriaceae were easily visible at 48 hours (Fig. 7). At the cooler temperatures Gram-positive cocci required up to 72 hours to be readily visible to the untrained eye. Periods of refrigeration at $4^{\circ} \mathrm{C}$. for up to 120 hours before incubation at $37^{\circ} \mathrm{C}$. had no effect on the final outcome.

Interpretation.-Negative plates and those with a light or mixed growth can be confidently discarded. A heavy pure growth (greater than about 300 colonies per final block) is provisionally designated as positive. Identification and antibiotic sensitivity testing can be performed from the growth, and, when indicated clinically, treatment can be started without further investigation. An equivocal result, or a positive result without clinical indication of infection, is reinvestigated either by a second screening test or, preferably, by routine laboratory methods before instituting treatment.

\section{Discussion}

The necessity for a simple screening method for the diagnosis and follow-up of urinary tract infections in hospital and in general practice is well recognized (Brit. med. F., 1965). For routine laboratory practice most authors regard semiquantitative methods, such as the standard loop methods (Hoeprich, 1960 ; O'Sullivan et al., 1960 ; Guttmann and Stokes, 1963), as satisfactory substitutes for potentially more accurate but timeconsuming dilution methods. Non-quantitative and uninterpreted methods are no longer acceptable (Bradley and Little, 1963).

Chemical methods for detecting significant bacteriuria' such as the triphenyl tetrazolium chloride test (Simmons and Williams, 1962) or the Griess nitrite test (Smith et al., 1961) have been used with variable success. In the hands of its authors the former showed $95 \%$ of infections (Simmons and Williams, 1962), but in another series only $44 \%$ of infections in the $10^{5}-10^{9}$ organisms per $\mathrm{ml}$. of urine range were detected (Hinton and van der Hoeven, 1965). The latter test is apparently even less satisfactory (Kincaid-Smith et al., 1964).

Whatever their accuracy chemical reactions reveal only that significant numbers of organisms are present. It is possible to obtain a positive result from a contaminated specimen, and while proper midstream specimens are desirable they are not always obtained. It is therefore important to be able to differentiate between contamination and infection. If a positive chemical test is obtained another specimen must be examined by conventional methods to identify the responsible organism (essential for proper follow-up studies) and to determine its antibiotic sensitivity pattern.

Cultural screening methods overcome these disadvantages of chemical tests. Methods such as the Leigh and Williams (1964) blotting-paper strip method, the dip-inoculum transport medium of Mackey and Sandys (1965), or the dip-slide method of Guttmann and Naylor (1967) require special equipment, admittedly minor in the blotting-paper method, and expert interpretation.

As the swab-plating method is satisfactory over a relatively wide temperature range it can be used where bacteriological incubators are not available, so long as the room is reasonably temperate. Keeping the plates at room temperature is preferable to incubating them in an airing or similar warm cupboard, as the heat tolerance of members of the Enterobacteriaceae above $37^{\circ} \mathrm{C}$. is far less 
than at a temperature below this. Thus, from general practice, the inoculated plate can be sent to the laboratory for incubation and interpretation. If this is impossible the plates can be kept by the practitioner and only " positives" submitted for identification and sensitivity testing. Unfortunately organisms causing infections in other situations are much more fastidious in their growth requirements, and it is not possible, therefore, to use modifications of this method for other bacteriological investigations.

MacConkey plates were used because they support the growth of virtually all organisms responsible for acute urinary tract infections, and they inhibit the swarming of Proteus species. They can be obtained pre-poured from commercial sources and do not deteriorate during 7 to 10 days at room temperature, especially if kept in plastic bags.

Other commercial cotton-wool swabs could doubtless be used equally well, but would require initial calibration with more accurate methods. Serum-coated swabs, being water-repellent, are unsuitable.

Though $10^{5}$ organisms per $\mathrm{ml}$. of urine has come to be regarded by many as the figure below which urinary infection is not present this is not always so. As was pointed out in Kass's (1955) original paper one of the circumstances in which, with an active urinary infection, fewer organisms are found is when there is frequency of micturition. There is then insufficient incubation time in the bladder for organisms to reach $10^{5} / \mathrm{ml}$. of urine. In general practice there is often "built-in" frequency, as many patients micturate immediately before leaving home. Specimens of urine obtained in these circumstances may therefore have fewer than $10^{5}$ organisms per $\mathrm{ml}$. in the presence of infection. Diuresis also significantly reduces the number of organisms (Roberts et al., 1967). Other less well recognized factors, such as the excretion of inhibitory substances from the diet, may also account for fewer than $10^{5}$ organisms per $\mathrm{ml}$. in the presence of infection. These factors must be considered in reading screening tests, just as they are in the interpretation of routine cultures. It is not necessarily of any disadvantage, therefore, that accurate counts between 250 and 500 colonies per inoculum are not possible. Indeed, it is questionable whether the rigid application of accurate methods is justifiable on such variable material. This can sometimes be undoubtedly misleading.

\section{Summary}

A cultural method for the investigation of urinary tract infections using commercially available cotton-wool swabs to inoculate MacConkey plates with freshly voided urine is described. This is simple to use, and does not necessaril require incubation at $37^{\circ} \mathrm{C}$. Cultural methods have advantage: over chemical screening tests, but some described previously require special equipment and thus are an added burden of laboratory preparation facilities.

We wish to acknowledge the co-operation of Dr. Betty Bass ir the part of the investigation carried out in general practice and of Sister E. F. Williams, of the Gynaecological Outpatient Departmen1 at the Royal Free Hospital, and the technical assistance of Mrs J. Wood, B.Sc.

Disposable swabs were obtained from Grahams Medical Product: Ltd., St. Jude Works, Westmoor Street, London S.W.7. Pre-pourec MacConkey plates can be obtained from Oxoid Ltd., London S.E.1

\section{REFERENCES}

Bradley, J. M., and Little, P. J. (1963). Brit. med. F., 2, 361.

Brit. med. $7.1965,1,605$.

Cruickshank, R. (editor) (1965). Medical Microbiology, 11th ed., p. 870 London.

Guttmann, D., and Naylor, G. R. E. (1967). Brit. med. F., 3, 343.

Iind Stokes, E. J. (1963). Ibid., 1, 1384.

Hinton, N. A., and van der Hoeven, E. (1965). Canad. med. Ass. 7., 93 639.

Hoeprich, P. D. (1960). F. Lab. clin. Med., 56, 899.

Kass, E. H. (1955). Amer. F. Med., 18, 764.

Kincaid-Smith, P., Bullen, M., Mills, J., Fussel, U., and Huston, $N$ (1964). Lancet, 2, 61.

Leigh, D. A., and Williams, J. D. (1964). 7. clin. Path., 17, 498.

Mackey, J. P., and Sandys, G. H. (1965). Brit. med. J., 2, 1286.

Mond, N. C., Percival, A., Williams, J. D., and Brumfitt, W. (1965) Lancet, 1, 514.

O'Sullivan, D. J., FitzGerald, M. G., Meynell, M. J., and Malins, J. M (1960). f. clin. Path., 13, 527.

Roberts, A. P., Robinson, R. E., and Beard, R. W. (1967). Brit. med. 9 1,400 .

Simmons, N. A., and Williams, J. D. (1962). Lancet, 1, 1377.

Smith, L. G., Thayer, W. R., Malta, E. M., and Utz, J. P. (1961). Ann intern. Med., 54, 66.

\title{
Blood Loss Using the Modified Two-layer Kiil Dialyser
}

\author{
D. B. EVANS,* M.B., M.R.C.P. ; E. M. CLARKSON, $\dagger$ B.SC. ; J. R. CURTIS, $\ddagger$ M.B., M.R.C.P.
}

Brit. med.F., 1967, 4, 651-653

One of the factors contributing to the anaemia of patients undergoing intermittent haemodialysis is blood loss due to blood remaining in the dialyser and extracorporeal circuit at the end of dialysis.

The present study was undertaken to determine the volume of blood remaining in the dialyser and arterial and venous blood lines at the end of dialysis, and to compare the efficiency of various techniques used to rinse back the blood in the dialyser and blood lines into the patient via the venous cannula at the end of dialysis.

\section{Methods}

\section{Methods of Determining Volume of Blood Remaining in Dialyser and Blood Lines at End of Dialysis}

A sample of the patient's blood was taken into a Sequestrene tube at the end of dialysis. Suitably diluted, this served as a standard with which to compare the haemoglobin concentration in the wash solution obtained from the dialyser and blood lines.

When dialysis had been discontinued the blood lines and dialyser were washed through with tap-water until the washings were colourless. The dialyser was then dismantled and any blood remaining on the cuprophane membranes was washed off with tap-water. All washings were collected and the total volume was measured. After careful mixing, an aliquot from the washings was taken and stored at $-15^{\circ} \mathrm{C}$. or $+4^{\circ} \mathrm{C}$. with the post-dialysis blood sample from the patient until the haemoglobin concentrations were estimated. Then $0.2 \mathrm{ml}$. of wellmixed whole blood from each patient was diluted to $50 \mathrm{ml}$. with $0.05 \%$ ammonia. Any intact red cells remaining in the wash solution were lysed by the addition of a small amount of

\footnotetext{
* Senior Medical Registrar, Renal Unit, Addenbrooke's Hospital, Cam-

+ Biochemist, Charing Cross Hospital Medical School, Fulham Hospital London.

‡Lecturer in Medicine, Charing Cross Hospital Medical School, Fulham Hospital, London.
} 